\title{
Analysis and Design of SIW Components Based on H-Plane Planar Circuit Approach
}

\author{
Isao Ohta and Mitsuyoshi Kishihara* \\ University of Hyogo \\ Japan \\ *Okayama Prefectural University \\ Japan
}

\section{Introduction}

A waveguide whose sidewalls are replaced with densely arranged metallic posts has been proposed. This guide enables the easy realization of circuit patterns by the arrangement of metallic posts periodically in a parallel-plate waveguide or a grounded dielectric substrate. This type of waveguide is called the post-wall waveguide (PWW) (Hirokawa \& Ando, 1998;Ando et al., 1998) or the substrate integrated waveguide (SIW) (Wu, 2001; Deslandes \& $\mathrm{Wu}, 2005)$. This SIW technology is applied to a feed waveguide for a slot array antenna, or a leakage wave antenna. In particular, recently, for the purpose of making use of merits such as low loss, low cost, and high-density integration of microwave and millimeter-wave components and subsystems, a SIW short-slot $90^{\circ}$ hybrid coupler, and a six-port receiver consisting of the $90^{\circ}$ couplers and/or power dividers have been developed. Since this technology is a relatively new concept, it is desired that more SIW circuit components and subsystems appear to open a new vista (Xu et al., 2005; Moldovan et al., 2006).

With regard to the analytical method of the SIW structure, the derivation of the propagation constant for the straight section of the guide has been studied on the basis of the Galerkin's method of moment (Hirokawa \& Ando, 1998). Then widths of the SIW structure that is equal to the cutoff frequency of the rectangular waveguide with perfectly metalized sidewalls have been obtained. Also, empirical equations for the equivalent widths have been proposed through experiments and simulations (Xu \& Wu, 2005; Cassivi et al., 2002). In (Xu $\& \mathrm{Wu}, 2005)$, the FDTD method and the multimode calibration method are used to analyze the dispersion characteristics of the complex propagation constants of the SIW structure. However, in the case of designing and analyzing the circuit components for practical use, a full-wave em-simulator (Ansoft HFSS) has been employed (Moldovan et al., 2006). Since the simulation requires a relatively long computing time, it is desirable to develop a faster solver for the optimization requiring recursive computations.

In this study, an analysis of the SIW structure is attempted by applying the analytical technique of the H-plane waveguide discontinuities based on the planar circuit approach (Kishihara et al., 2006; Kishihara et al., 2004). This technique can reduce the computation 
time considerably, because the two-dimensional structure of the SIW is well used to advantage in the analysis.

The present two-dimensional approach is about 10 times faster than the full-wave simulator (HFSS). First, a planar-circuit model of the SIW for analysis is introduced. The analytical procedure consists of 1) the derivation of the mode impedance matrices for regular-shaped planar circuits and 2) short-circuiting of fictitious ports arranged on the peripheries of the metallic posts, in accordance with the treatment of the H-plane waveguide discontinuities containing metallic obstacles. In the SIW structure, a leakage field problem outside the guide occurs due to the gaps of the arrayed metallic posts. This phenomenon should be excluded in the practical design of the passive circuit components, except in cases where it is used positively, such as in leakage wave antennas. In this paper, the leakage field is considered by connecting fictitious TEM transmission lines on the periphery of the planar circuit model and terminating them with their characteristic impedances.

Next, the S-parameters of the SIW straight section are calculated as a numerical example. In addition, a situation in which radiation is produced outwards from the guide is prepared intentionally by placing metallic posts at slightly broader intervals, and the validity of the above-mentioned treatment is examined. Then, the propagation constant of the SIW is calculated using the H-plane planar circuit approach along with the TRL calibration technique for a vector network analyzer (Pozar, 1998). In the analysis, the reduction of the computational time is achieved by utilizing the periodic structure of the SIW.

In this work, the present method is applied to the design of two types of SIW corners, a right-angled circular corner and a right-angled corner with a cylindrical region of air (an airpost). The corners are constructed of arrayed metallic posts similarly to an SIW straight-line section. For the corner with one air-post, a portion of the dielectric is replaced with air to obtain a matched state. The validity of the analysis and the design results are confirmed using an em-simulator (HFSS).

Moreover, a cruciform SIW quadrature hybrid (Ohta et al., 2007) is designed based on the idea similar to an H-plane crossed-waveguide quadrature 3-dB hybrid (Toda et al., 2006). The SIW has a planar structure parallel to the plane of magnetic field, and the electromagnetic field in the circuit dose not change in the direction perpendicular to the magnetic plane (H-plane). This implies that the design concept and analytical method of the SIW circuits follow those of the H-plane planar circuit (Kishihara et al., 2006; Kishihara et al., 2004). In the analysis, reduction of the computational time is successfully achieved by utilizing the periodic structure of the SIW. Finally, optimum design of crossed-SIW quadrature $3-\mathrm{dB}$ hybrids is described. Good hybrid properties are obtained for some design frequencies.

\section{Planar circuit model and analytical procedure}

Figure 1 exhibits a portion of the SIW straight-line section. Dielectric material with relative permittivity $\varepsilon_{r}$ is filled between the top and bottom metallic plates, and metallic posts of radius $r$ are placed at width $a_{f}$ and spacing $s$. Generally, since the height of the SIW is much less than the wavelength used in its circuit system, the electromagnetic field is constant in the height direction. Therefore, the propagation and non-propagation modes excited in the SIW are $\mathrm{TE}_{n 0}$-like modes, which are very similar to the $\mathrm{TE}_{n 0}$ modes of the conventional waveguide, and hence the electric field consists of only a vertical component. In addition, 
Fig. 1. Structure of SIW straight-line section
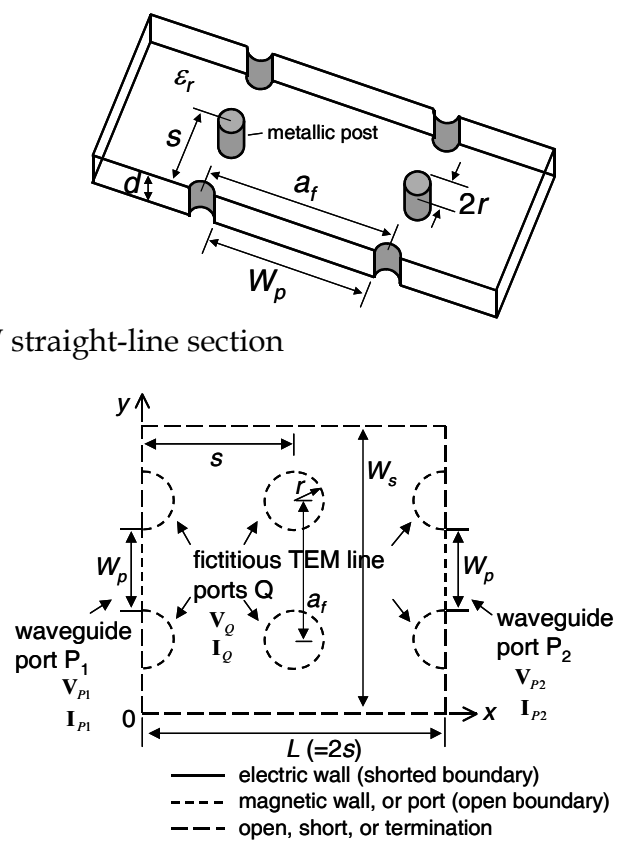

Fig. 2. Planar circuit model for SIW straight-line section

the leakage loss from the gaps between the side-wall posts is very low, because the surface current of $\mathrm{TE}_{n 0}$-like modes flows parallel to the metallic posts of the side-wall. In particular, if the SIW is excited by the $\mathrm{TE}_{10}$ (or $\mathrm{TE}_{n 0}$ ) mode of a rectangular waveguide with the same height and dielectric constant as the substrate, then the electromagnetic field in the circuit never changes in the vertical direction because the structure possesses no variation along the same direction. In other words, we can describe the circuit performance using only $\mathrm{TE}_{n 0}$-like modes. From this fact, in this study, we apply the H-plane planar circuit approach to analyze the SIW circuit system.

Figure 2 illustrates the planar circuit model of the SIW straight section corresponding to that in Fig.1. A rectangular area of width $W_{s}\left(>4 r+W_{p}\right)$ and length $L(=2 s)$ extended outside the posts is considered in order to treat the leakage field distribution. The solid and the dotted lines represent the electric wall (shorted boundary) and the magnetic wall (open boundary), respectively. For the periphery of the planar circuit depicted by the broken line, any boundary condition of open, shorted, or terminated with characteristic impedances can be used. It is possible to carry out the analysis with an open or shorted boundary if the influence of radiation is negligible. However, in the case that radiation losses must be taken into account, the leakage waves from the gaps of the side-wall posts should be absorbed. In this paper, we attempt to absorb the waves by fictitiously connecting many TEM parallelplate lines of narrow width to the boundaries of the rectangular planar circuit and teminating the fictitious ports with their characteristic impedances.

The input/output waveguide ports with a width of $W_{p}$ are located at the left and right sides of the circuit (ports $P_{1}$ and $P_{2}$ ). On the peripheries of the metallic posts, moreover, the above fictitious TEM-line ports are arranged close together without any gaps (ports $Q$ ). $\mathbf{V}_{i}$ and $\mathbf{I}_{i}$ 
stand for the voltage and current vectors of the $i$ th port $\left(i=P_{1}, P_{2}\right.$, or $\left.Q\right)$. Deriving the mode impedance matrices between the input/output ports $\left(P_{1}, P_{2}\right)$ and the fictitious ports $(Q)$ by the planar circuit approach, and short-circuiting only the fictitious ports arranged on the peripheries of the metallic posts, one can obtain the 2-port mode impedance matrix containing the characteristics of the arrayed posts. Therefore, the scattering parameters can be calculated.

On the basis of the planar circuit approach or the impedance Green's function approach, the voltage at any point on the planar circuit can be written as

$$
V(x, y)=j \omega \mu_{0} d \iint_{D} G\left(x, y \mid x_{0}, y_{0}\right) J\left(x_{0}, y_{0}\right) d x_{0} d y_{0},
$$

where $J\left(x_{0}, y_{0}\right)$ denotes the source current normally injected into the circuit, and a Green's function $G()$ must satisfy the boundary conditions of the planar element. $D$ denotes the twodimensional region of the planar circuit. By expanding the fields in the input and output ports in terms of eigenmodes of the rectangular waveguide, the mode impedance matrix can be given as

$$
Z_{p, q}^{i, j}=\frac{j \omega \mu_{0} d}{W_{i} W_{j}} \int_{0}^{W_{i}} \int_{0}^{W_{j}} G\left(x_{i}, y_{i} \mid x_{j}, y_{j}\right) f_{i, p}\left(s_{i}\right) f_{i, q}\left(s_{j}\right) d s_{i} d s_{j},
$$

where $W_{i}$ and $W_{j}$ indicate the widths of the $i$ th and $j$ th coupling waveguide ports, respectively, and $f_{i, p}$ represents the eigenfunction of the waveguide with shorted or open boundaries and is given as

$$
f_{i, p}\left(s_{i}\right)=\left\{\begin{array}{c}
\sqrt{2} \sin \left(\frac{p \pi}{W_{i}} s_{i}\right) \text { for shorted boundaries } \\
\varepsilon_{p} \cos \left(\frac{p \pi}{W_{i}} s_{i}\right) \text { for open boundaries, }
\end{array}\right.
$$

where the origin of argument $s_{i}$ is assigned to one side of the coupling waveguide port. $s_{i}$ varies from 0 to $W_{i}$, and the integration in Eq.(2) is over the width of the port. Therefore, the $x y$-coordinate values must be transformed to the local coordinate value $s_{i}$ or $s_{j}$ of the coupling port. In Eq. (3), the sine function corresponds to the usual $\mathrm{TE}_{p 0}$ modes of the rectangular waveguide with shorted boundaries. The cosine function is applied to the ports with open boundaries. $\varepsilon_{p}$ is 1 for $p=0$ and $\sqrt{2}$ for $p \geq 1$. The cosine function is applied to the fictitious ports in this work, though only the fundamental TEM mode $(p=0)$ is considered because of the sufficiently narrow port-width. The suffixes $i$ and $p$ denote the port and mode numbers, respectively. $W_{i}$ is the width of the $i$ th port. Green's function can be expanded in terms of eigenfunctions of the corresponding rectangular planar circuit.

$$
\begin{gathered}
G\left(x_{i}, y_{i} \mid x_{j}, y_{j}\right)=-\frac{1}{L W_{s}} \sum_{m=0}^{\infty} \sum_{n=0}^{\infty} \frac{\psi_{m, n}\left(x_{i} y_{i}\right) \psi_{m, n}\left(x_{j}, y_{j}\right)}{k^{2}-\left(\frac{m \pi}{L}\right)^{2}-\left(\frac{n \pi}{W_{s}}\right)^{2}} \\
\psi_{m, n}=\varepsilon_{m} \varepsilon_{n} \cos \frac{m \pi}{L} x \cos \frac{n \pi}{W_{s}} y
\end{gathered}
$$

Equation (5) is applicable for the segments with open boundaries. $\varepsilon_{m}$ is 1 for $m=0$ and $\sqrt{2}$ for $m \geq 1$.

Now, we define the mode impedance matrix of the rectangular segment between two ports, $P_{1}$ and $P_{2}$, and fictitious ports $Q$ as

$$
\left[\begin{array}{c}
\mathbf{V}_{P} \\
\mathbf{V}_{Q}
\end{array}\right]=\left[\begin{array}{ll}
\mathbf{Z}_{P P} & \mathbf{Z}_{P Q} \\
\mathbf{Z}_{Q P} & \mathbf{Z}_{Q Q}
\end{array}\right]\left[\begin{array}{l}
\mathbf{I}_{P} \\
\mathbf{I}_{Q}
\end{array}\right]
$$


where abbreviations $\mathbf{V}_{P}=\left\{\mathbf{V}_{P 1}^{t}, \mathbf{V}_{P 2}^{t}\right\}^{t}$ and $\mathbf{I}_{P}=\left\{\mathbf{I}_{P 1}{ }^{t}, \mathbf{I}_{P 2}\right\}^{t}$ are used. If the fictitious ports are assumed only for the metallic posts, ports $Q$ are short-circuited by enforcing the boundary condition $\mathbf{V}_{Q}=0$ on the peripheries of the metallic posts. Substituting it into Eq.(6) gives the 2-port mode impedance matrix involving the discontinuity effects of the posts.

$$
\mathbf{Z}_{P P}{ }^{\prime}=\mathbf{Z}_{P P}-\mathbf{Z}_{P Q} \mathbf{Z}_{Q Q}{ }^{-1} \mathbf{Z}_{Q P}
$$

Finally, by terminating the higher order modes in ports $P_{1}$ and $P_{2}$ with their characteristic impedances, the 2-port impedance matrix for the $\mathrm{TE}_{10}$ mode can be obtained.

Furthermore, when the leakage field outside the posts must be considered, fictitious TEM lines are connected to the periphery of the planar circuit and terminated with impedances. The fictitious TEM lines are sufficiently narrow ports with open-boundary sidewalls. In this paper, we assume a dielectric substrate that extends infinitely outside the rectangular region. Then, the leakage field from the SIW structure is considered to be a TEM mode propagating in the parallel-plate waveguide. On the basis of circuit theory, no reflection occurs at the junction between a TEM line of $1 \Omega$ and $N$ TEM lines of $N \Omega$. This means that the outgoing waves branch into the narrow TEM lines without reflection, if sufficiently narrow TEM lines are arranged on the periphery without gaps. Therefore, the fictitious TEM lines ought to be terminated with their characteristic impedances to suppress the reflections of the outgoing waves. Even if the transversely changing fields arrive at the periphery, their field distributions can be approximated by the sufficiently narrow TEM ports, because the field is considered constant in the narrow width. In the present treatment, only the leakage waves normal to the boundaries are absorbed, such as Mur's first-order absorbing boundary condition in the FDTD method. The leakage waves can be decomposed into the field components propagating along the $x$ - and $y$-directions. At the boundaries $y=0$ and $W_{s}$, the leakage waves propagating along the $y$-direction are absorbed. The remaining components propagating along the $x$-direction are absorbed at the boundaries $x=0$ and $L$. In the numerical calculation, the areas that do not affect the field distribution of the main propagation mode extending slightly outside the posts and that attenuate evanescent waves should be considered. Namely, a length of about $\lambda g / 4$ or more should be considered outside the posts. Then it is expected that only the TEM wave reaches the periphery.

Consequently, by deriving the mode impedance matrices among the input/output ports, the fictitious ports on the metallic posts, and the fictitious TEM lines on the periphery of the circuit, and short-circuiting and terminating the fictitious ports and TEM lines respectively, we can obtain the impedance matrix of the SIW structure containing the leakage field effects.

\section{Numerical results}

\subsection{Straight section}

The S-parameters of the SIW structure shown in Fig.1 are computed to demonstrate the usefulness of the present analytical method. The dimensions of the guide are chosen as $\varepsilon_{r}=2.17, d=1.52 \mathrm{~mm}$, and $r=0.30 \mathrm{~mm}$. The spacing $s$ and the width $a_{f}$ of the posts are varied in pairs as $\left(s, a_{f}\right)=(1.00 \mathrm{~mm}, 4.92 \mathrm{~mm}),(1.50 \mathrm{~mm}, 4.85 \mathrm{~mm})$, and $(2.00 \mathrm{~mm}, 4.77 \mathrm{~mm})$ after Ref. (Hirokawa, 1998). The SIW structure and the field distribution simulated using the HFSS at $40 \mathrm{GHz}$ are depicted in Fig.3(a), and the frequency characteristics of the $S$-parameters are shown in Fig.3(b). In the calculation, the width of the fictitious TEM lines at the circumference of the rectangular planar circuit was chosen to be $0.1 \mathrm{~mm}$, and 10 fictitious TEM lines on the peripheries of each metallic post were considered. The fictitious ports were 


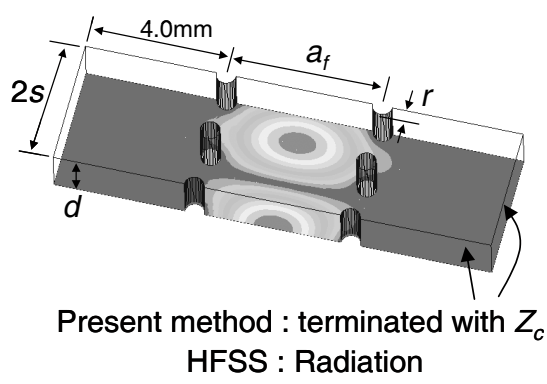

(a)

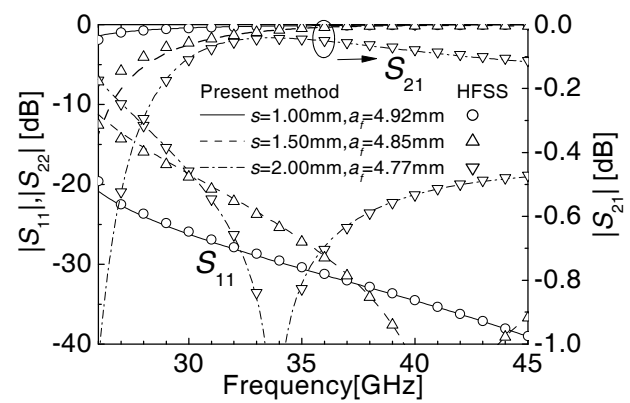

(b)

Fig. 3. (a) Structure and field distribution for the SIW straight section and (b) the frequency characteristics of the $S$-parameters

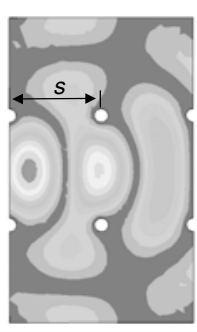

(a)

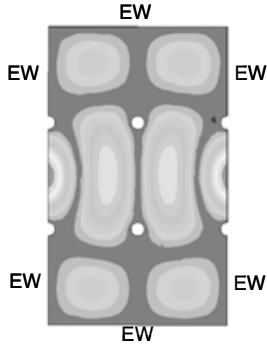

(b)

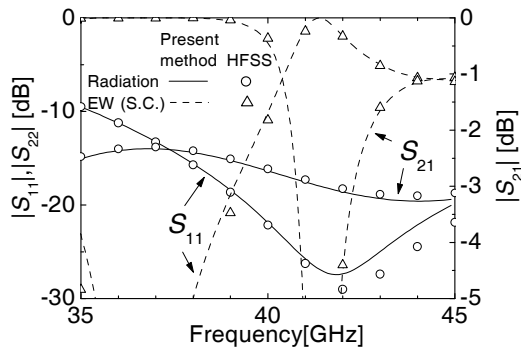

(c)

Fig. 4. Treatment of leakage field. (a) Structure and field distribution simulated using HFSS (boundary: radiation). (b) Structure and field distribution simulated using HFSS (boundary: electric wall). (c) Frequency characteristics of the $S$-parameters

arranged without gaps. For the doubly infinite series of $m \times n, 100 \times 100$ modes were considered. In each exciting waveguide, 8 modes $\left(\mathrm{TE}_{p 0} ; p=1,2, \ldots, 8\right)$ were considered. The results simulated using the HFSS are also plotted for comparison. In the present analysis, the regions of $4.0 \mathrm{~mm}$ in length $\left(\cong 0.65 \lambda_{\mathrm{g}}\right.$ at $\left.40 \mathrm{GHz}\right)$ are considered outside the arrayed posts. The periphery of the planar circuit is terminated with the characteristic impedances of the fictitious TEM lines. The computation time at one frequency point is 11 seconds for $s=2.00 \mathrm{~mm}$ and $a_{f}=4.77 \mathrm{~mm}$, while the HFSS requires 147 seconds (frequency $=37 \mathrm{GHz}$, adaptive pass=8, Delta $S<0.001$ ) on a Pentium4 3.2 GHz PC. The present method requires about $1 / 13$ of the computation time compared with the HFSS. The two results are in good agreement and the validity of the analysis is confirmed.

Next, the validity of the treatment of the leakage field is examined by widening the spacing $\mathrm{s}$ to $4.0 \mathrm{~mm}$. Fig.4(a) shows the field distribution calculated using the HFSS (at $40 \mathrm{GHz}$ ). The $\mathrm{TE}_{10}$ mode is incident from the left side of the guide. It is clear that the field is leaking. When the periphery is assigned to electric walls, a resonant mode appears, as shown in Fig.4(b). The $S$-parameters obtained by the present method and the HFSS for these boundary conditions are shown in Fig.4(c). The solid and the broken lines represent the terminated and the short-circuited results for the present method, respectively. The markers are those for the HFSS. 


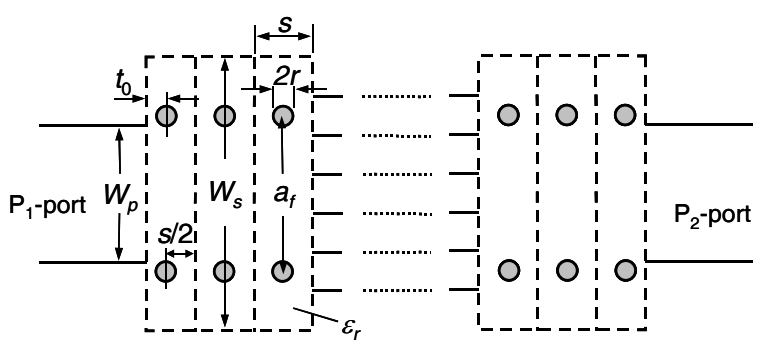

(a)

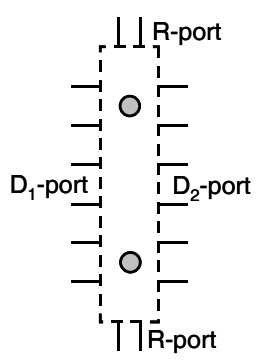

(b)

Fig. 5. Analytical procedure for SIW straight-line. (a) Division of SIW line into periodic structures. (b) One-period section

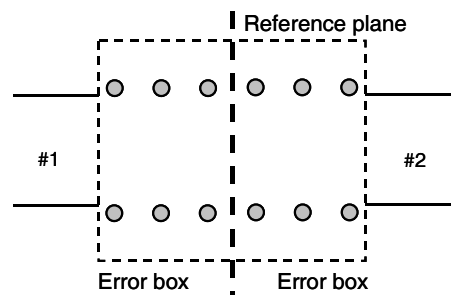

(a)

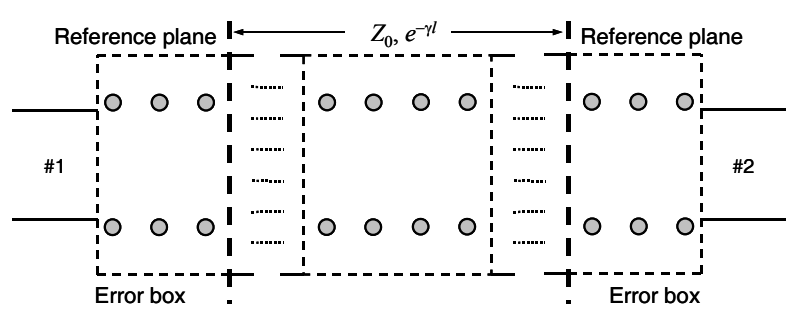

(b)

Fig. 6. Thru and Line connections. (a) Thru. (b) Line

It is found that $S_{21}$ in the solid line indicates approximately $-3 \mathrm{~dB}$ by radiation, while the results shown by the broken lines show completely different characteristics because of the short-circuited periphery. Both these results agree well with the results obtained with the HFSS. The present treatment of the leakage field is verified.

\subsection{Propagation constant of SIW line}

The propagation constant of the SIW line can be derived using the above technique. The straight-line has a periodical structure, except for the exciting parts at the two ends, as shown in Fig.5 (a), where a slightly modified planar circuit model is employed. The semicircular posts are eliminated and the one-period section in Fig.5 (b) is considered. In order to reduce the scale of the analysis, we first compute the mode impedance matrices of the exciting structure with the rectangular waveguide and one-period section, then use the segmentation method (Chadha \& Gupta, 1981) one after the other, and finally derive the mode impedance matrix between the two exciting waveguides. Moreover, by terminating the higher-order $\mathrm{TE}_{p 0}$ modes of the two rectangular waveguides with their characteristic impedances, we can obtain the two-port impedance matrix, and hence the scattering matrix for the $\mathrm{TE}_{10}$ mode. However, in the strict sense, the resultant matrix is not that of the SIW, because it contains some vagueness of the excitation region.

For that reason, the TRL calibration technique (Pozar, 1998) used in the measurement with a vector network analyzer is applied, though the "Reflect connection" is unnecessary in this case. Now, if it is assumed that the two-port scattering matrices for the "Thru" and "Line" connections shown in Figs.6 (a) and 6 (b) are given as 


$$
\left[\begin{array}{l}
b_{1} \\
b_{2}
\end{array}\right]=\left[\begin{array}{ll}
T_{11} & T_{12} \\
T_{21} & T_{22}
\end{array}\right]\left[\begin{array}{l}
a_{1} \\
a_{2}
\end{array}\right]
$$

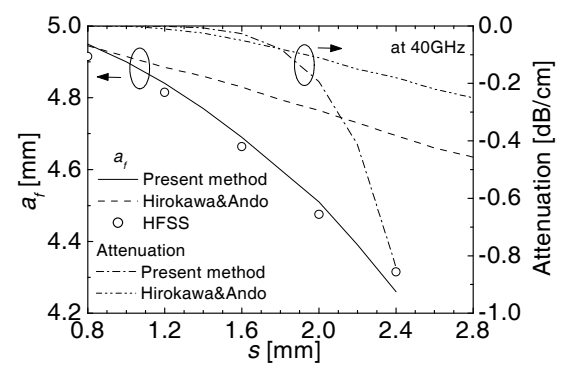

Fig. 7. Equivalent SIW width $a_{f}$ and attenuation coefficient as a function of post spacing $s$. $\left(\varepsilon_{r}=2.17, d=1.52 \mathrm{~mm}, r=0.30 \mathrm{~mm}\right)$

$$
\left[\begin{array}{l}
b_{1} \\
b_{2}
\end{array}\right]=\left[\begin{array}{ll}
L_{11} & L_{12} \\
L_{21} & L_{22}
\end{array}\right]\left[\begin{array}{l}
a_{1} \\
a_{2}
\end{array}\right],
$$

respectively, then the propagation term of the SIW straight-line with length $l$ can be derived as follows:

$$
\begin{aligned}
& e^{-\gamma}=\frac{\kappa \pm \sqrt{\kappa^{2}-4 L_{12}^{2} T_{12}^{2}}}{2 L_{12} T_{12}} \\
& \kappa=L_{12}^{2}+T_{12}^{2}-\left(T_{11}-L_{11}\right)^{2} .
\end{aligned}
$$

Thus, we can derive the propagation constant $\gamma$ of the SIW, excluding the error of the exciting parts, under the condition that the SIW sustains only its dominant mode ( $\mathrm{TE}_{10}$-like mode).

Figure 7 shows the equivalent SIW width $a_{f}$ and the attenuation coefficient as a function of the post spacing $s$ to obtain the equal phase constant ( $=1.206 k_{0}, 1011 \mathrm{rad} / \mathrm{m}$ at $40 \mathrm{GHz}$ ) of the rectangular waveguide of width $a_{e}=4.43 \mathrm{~mm}$ (Hirokawa, 1998) $(r=0.30 \mathrm{~mm}, d=1.52 \mathrm{~mm}$, $\left.\varepsilon_{r}=2.17, W_{s}=a_{f}+2 r+4.00 \mathrm{~mm}, t_{0}=r+0.01 \mathrm{~mm}, W_{p}=a_{e}\right)$. The results obtained by the present method are compared with those in Ref. (Hirokawa \& Ando, 1998). It is noted that when $s$ increases, $a_{f}$ of the present method decreases rapidly. In order to verify the calculation results, the field distribution simulated using the HFSS is used. $a_{f}$ can be estimated by measuring the guide wavelength. The markers in Fig.7 are those of the HFSS. The results of the present method agree well with those obtained with the HFSS. In Fig.7, the attenuation coefficient is also indicated. It is found that as the post spacing $s$ becomes larger, the attenuation increases sharper than that in Ref. (Hirokawa \& Ando, 1998). For a narrow post spacing up to about $1.8 \mathrm{~mm}$, the results show fair agreement.

Figures $8(\mathrm{a})$ and $8(\mathrm{~b})$ show the computed frequency dependences of the phase and the attenuation constants for $s=1.00 \mathrm{~mm}, 1.20 \mathrm{~mm}$, and $1.40 \mathrm{~mm}$. The phase constants (dispersion characteristics) agree well with those of the $\mathrm{TE}_{10}$ mode of the conventional rectangular waveguide (equivalent width $a_{e}$ is $4.43 \mathrm{~mm}$, which is calculated from the cutoff frequency of the SIW). The attenuation constants are less than $0.001 \mathrm{~Np} / \mathrm{m}, 0.01 \mathrm{~Np} / \mathrm{m}$ and $0.07 \mathrm{~Np} / \mathrm{m}$ for $s=1.00 \mathrm{~mm}, 1.20 \mathrm{~mm}$, and $1.40 \mathrm{~mm}$, respectively, at operation bands over 30 
GHz. It is recognized that reasonable low-loss properties are obtained for the narrow post spacing.

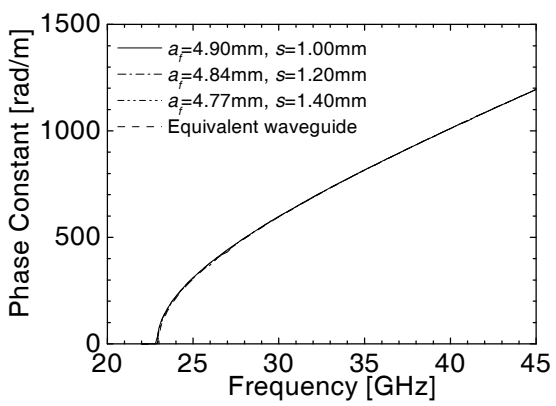

(a)

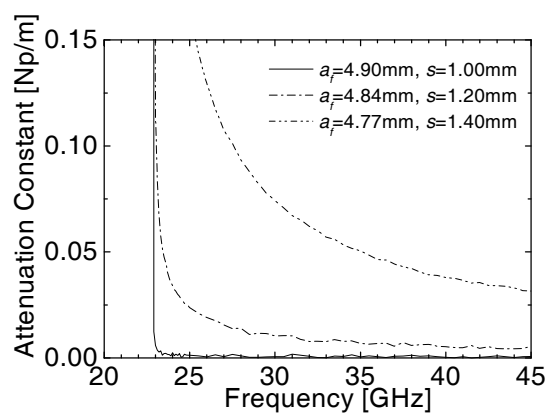

(b)

Fig. 8. Dispersion characteristics of the SIW TE $E_{10}$-like mode. (a) Phase constant. (b) Attenuation constant. $\left(\varepsilon_{r}=2.17, d=1.52 \mathrm{~mm}, r=0.30 \mathrm{~mm}\right)$

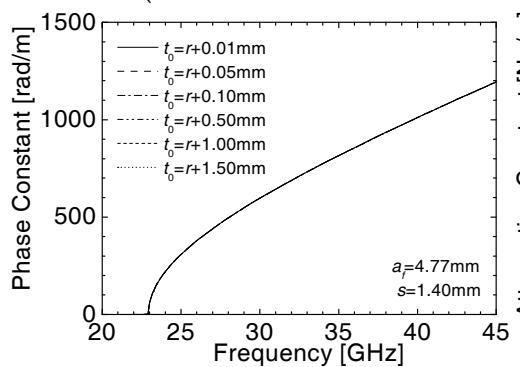

(a)

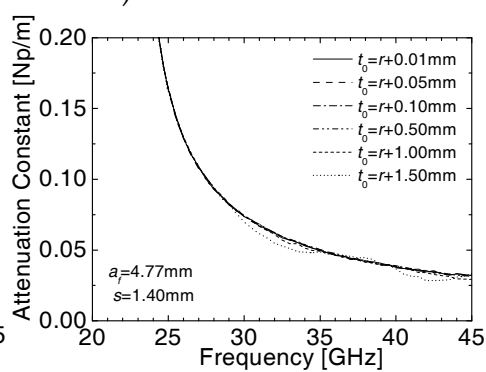

(b)

Fig. 9. Dispersion characteristics with various gap $t_{0}$ in the error boxes. (a) Phase constant. (b) Attenuation constant. $\left(\varepsilon_{r}=2.17, d=1.52 \mathrm{~mm}, r=0.30 \mathrm{~mm}\right)$

In order to verify that the characteristics of the exciting ports are properly eliminated, the influence of gap $t_{0}$ in the error boxes is examined. Figures $9(\mathrm{a})$ and $9(\mathrm{~b})$ display the dispersion characteristics for $a_{f}=4.77 \mathrm{~mm}$ and $s=1.40 \mathrm{~mm}$ with gap $t_{0}$ varied from $r+0.01 \mathrm{~mm}$ to $r+1.50 \mathrm{~mm}$. All the phase constants in Fig.9(a) are consistent with each other. The attenuation constants in Fig.9(b) are in good agreement for $t_{0} \leq r+1.00 \mathrm{~mm}$. It is noted that the TRL calibration technique works well, though gap $t_{0}$ should be small to obtain accurate results. Particularly in the case of $t_{0}=r+1.50 \mathrm{~mm}$, the attenuation constant varies widely, because the attenuation at the gap is larger than that of the SIW line. When gap $t_{0}$ becomes large, excitation of the SIW results in failure.

Figures 10(a) and 10(b) also show the computed frequency dependences of the phase and attenuation constants of the SIW TE 10 -like mode. In this calculation, the dimensions of the guide are selected to be $\varepsilon_{r}=2.33, d=0.508 \mathrm{~mm}, r=0.40 \mathrm{~mm}, s=2.00 \mathrm{~mm}, a_{f}=7.20 \mathrm{~mm}, W_{s}=$ $a_{f}+2 r+4.00 \mathrm{~mm}, t_{0}=r+0.01 \mathrm{~mm}$, and $W_{p}=6.86 \mathrm{~mm}$ after Ref. (Xu \& Wu, 2005). The cutoff frequency is estimated to be $14.3 \mathrm{GHz}$ from Fig.10(a). At lower frequencies, the attenuation constant increases exponentially owing to radiation loss. The results of the present method are compared with those in Ref. (Xu \& Wu, 2005) derived from the multimode calibration 
method. The two results are in good agreement. Thus the validity of the numerical results is confirmed.

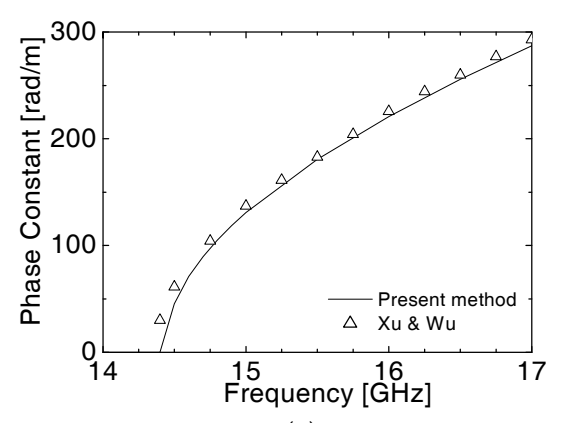

(a)

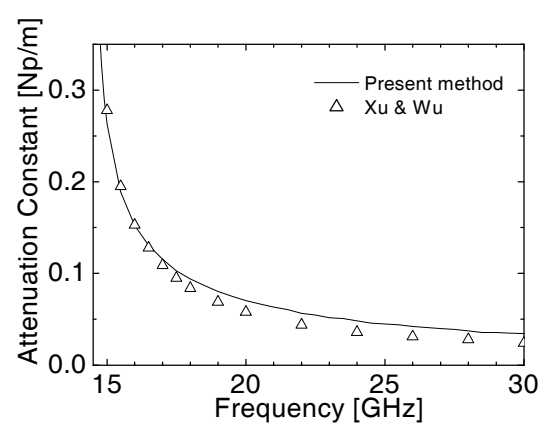

(b)

Fig. 10. Dispersion characteristics of SIW TE 10 -like mode. (a) Phase constant. (b) Attenuation constant. $\left(\varepsilon_{r}=2.33, d=0.508 \mathrm{~mm}, r=0.40 \mathrm{~mm}\right)$

In the calculation, the width of the fictitious TEM lines at the circumference of the rectangular planar circuit was chosen to be $0.1 \mathrm{~mm}(R$-port), and 10 fictitious TEM lines on the peripheries of each metallic post were considered. Moreover, we employed 16 modes $\left(\mathrm{TE}_{q 0} ; q=0,1,2, \ldots, 15, \mathrm{TE}_{00}\right.$ mode: TEM mode) of the waveguide with magnetic sidewalls of width $W_{s}$, which were shown by ports $D_{1}$ and $D_{2}$ in Fig.5(b), as fictitious connecting ports for uniting each periodic segment. In each exciting waveguide, 8 modes $\left(\mathrm{TE}_{p 0} ; p=1,2, \ldots, 8\right)$ were considered.

\section{Design of SIW components}

\subsection{SIW corners}

The present method is applied to the design of SIW corners to demonstrate the usefulness and flexibility of the method. Figure 11(a) shows a right-angled circular corner constructed from the post-wall waveguide, where the metallic posts are arranged in a circular form at intervals of $\Delta \theta$. Because there is no restriction in arranging the posts, any circuit configuration can be analyzed directly by the planar circuit approach. The dimensions of the guide are chosen to be $\varepsilon_{r}=2.17, d=1.52 \mathrm{~mm}$, and $r=0.30 \mathrm{~mm}$. Spacing $s=1.00 \mathrm{~mm}$, width $a_{f}$ $=4.90 \mathrm{~mm}$, and angle $\Delta \theta=11.25^{\circ}\left(a_{f} \Delta \theta=0.96 \mathrm{~mm}\right)$ are used to ensure low loss. Figure 11(b) indicates the frequency characteristics of the $S$-parameters. It is found that small insertion losses of less than $0.01 \mathrm{~dB}$ as well as the relatively low reflection characteristic of less than $25 \mathrm{~dB}$ are achieved at the operation band. The calculated results agree well with the results obtained with the HFSS.

Figure 12(a) shows the structure of a right-angled corner with one air post. The corner consists of the metallic posts arranged in a right-angle form. A portion of the dielectric material is replaced with an air region of radius $R$. If the dimensions and the position of the air region are optimized, a low reflection is expected. The analysis is performed by shortcircuiting fictitious ports for the arrayed posts, and by the desegmentation-segmentation process (Kishihara et al., 2006; Kishihara et al., 2004) for the air post region. Namely, the 
analytical procedure consists of the following three steps: 1) the derivation of the mode impedance matrix of the dielectric post (the same region as the air post), 2) the extraction of the dielectric post by desegmentation, and 3) the substitution of the air post by segmentation. Figure 14 shows the frequency characteristics of the $S$-parameters obtained by optimizing the radius $R$ and the position $c_{l}$ of the air post. Low reflections of less than $-30 \mathrm{~dB}$ are achieved around $c_{l}=4.12 \mathrm{~mm}$ and $R=1.16 \mathrm{~mm}$, though the bandwidth is relatively narrow. It is found that this right-angled circular corner exhibits low-reflection characteristics compared with the return losses of about $-10 \mathrm{~dB}$ to $0 \mathrm{~dB}$ of a right-angled corner without the air post. Figure 12(b) also shows the results obtained with the HFSS. The two results are in good agreement. The validity of the design results is confirmed.

\subsection{SIW cruciform quadrature hybrids}

In this section, we design crossed-SIW quadrature hybrids shown in Fig.13. The design frequencies are chosen in quasi-millimeter-wave regions. The dimensions of the SIW are chosen as $\varepsilon_{r}=2.17, d=0.508 \mathrm{~mm}, r=0.40 \mathrm{~mm}, s=1.80 \mathrm{~mm}$, and $a_{f}=6.72 \mathrm{~mm}$, because of the good propagation properties. The frequency dependences of propagation constant of the $\mathrm{SIW} \mathrm{TE}_{10}$-like mode are displayed in Fig.14. The attenuation constants are less than 0.03 $\mathrm{Np} / \mathrm{m}$ in the operation band of the SIW, and reasonable low loss properties are obtained. In addition, phase constants (dispersion properties) well agree with those of the $\mathrm{TE}_{10}$ mode for the conventional rectangular waveguide whose width $\left(a_{\mathrm{e}}=6.32 \mathrm{~mm}\right)$ is calculated from the same cutoff frequency as the SIW. In the design, we considered the square planar circuit of $W \times W$ with open boundary, which can divide into four one-port with regard to the two symmetry planes (Toda et al., 2006). The even-odd mode analysis is applied for the analysis of the SIW cruciform hybrid. Namely, the reflection coefficients of the four one-port are derived based on the planar circuit approach, and then the scattering parameters of the entire hybrid circuit are computed. The optimization of the circuit dimensions such as the radii and positions of the metallic posts in the cross junction and at the input/output ports

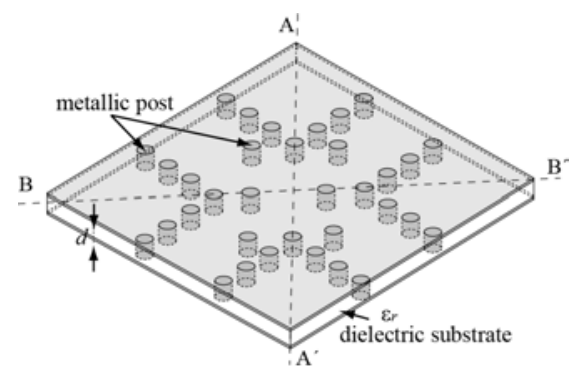

(a)

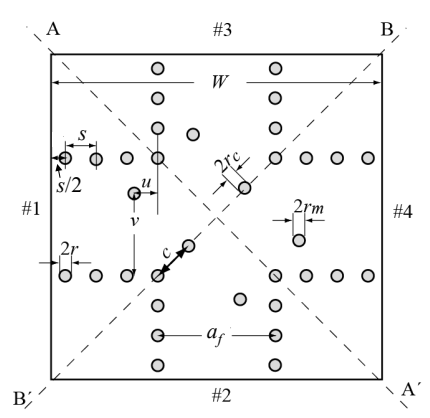

(b)

Fig. 13. Structure of SIW cruciform hybrid. (a) Sketch. (b) Plane figure

were performed using Powell's method (Powell, 1964) as a mathematical technique.

Figure 15 exhibits the scattering parameters of the hybrid designed at the center frequency of $24 \mathrm{GHz}$. The dots in the figure represent the simulation results using HFSS. Both the results exhibit good agreement with each other. It is shown that the H-plane planar circuit approach is a useful design tool for the SIW circuit components. The return loss and 
isolation are better than $20 \mathrm{~dB}$ in the bandwidth from 23.4 to $24.9 \mathrm{GHz}$, and the imbalance of the power split stays within $\pm 0.2 \mathrm{~dB}$ over the same bandwidth. Furthermore, we attempted to design at various center frequencies. Figure 16 (a) and (b) demonstrate the design results at 21 and $27 \mathrm{GHz}$, respectively. Although the bandwidth of the hybrid for the center frequency of $21 \mathrm{GHz}$ is narrow, the hybrid designed at $27 \mathrm{GHz}$ shows a relatively wide bandwidth of 26 to $32 \mathrm{GHz}$. The latter result suggests a possibility of realizing wider bandwidth by the use of widened cross-junction similarly to a crossed H-plane waveguide

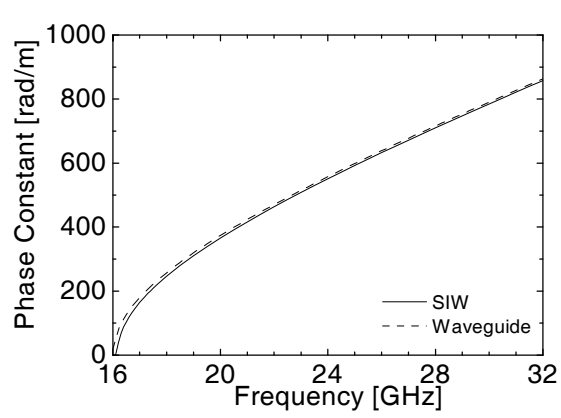

(a)

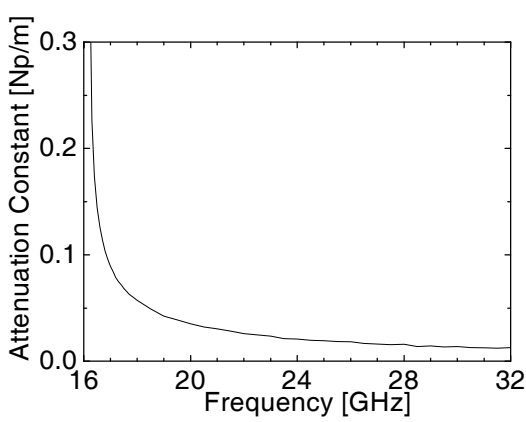

(b)

Fig. 14. Dispersion characteristics of SIW TE10-like mode for the SIW cruciform hybrids. (a) Phase constant. (b) Attenuation constant. $\left(\varepsilon_{r}=2.17, d=0.508 \mathrm{~mm}, r=0.40 \mathrm{~mm}, s=1.80 \mathrm{~mm}\right)$

hybrid (Toda et al., 2006).

\section{Conclusion}

A method of analyzing the SIW structure was demonstrated, in which the analytical technique of the H-plane waveguide discontinuities was applied on the basis of the planar circuit approach. The leakage field was considered by connecting fictitious TEM lines and terminating them with their characteristic impedances. The propagation constants of the SIW were calculated with the use of the TRL calibration technique. Moreover, the present method was applied to the design of the SIW corners. The validity of the numerical results was verified through comparison with the results in the references and those obtained with the HFSS. In addition, a novel cruciform SIW hybrid has been proposed, and some hybrids with good hybrid properties have been designed using the H-plane planar circuit approach. The design results exhibit good agreement with the simulation results (HFSS), and the validity of the design concept is confirmed. It has been shown that the H-plane planar circuit approach is one of effective analysis techniques for the SIW circuit components, because the metallic posts can be arranged in arbitrary positions.

\section{References}

Ando, M.; Hirokawa, J. ; Yamamoto, T. ; Akiyama , A. ; Kimura, Y. \& Goto, N. (1998). Novel single-layer waveguides for high-efficiency millimeter-wave arrays, IEEE Transactions on Microwave Theory and Techniques, Vol.46, No.6, pp. 792-799 
Cassivi, Y., Perregrini, L., Arcioni, P., Bressan, M., Wu, K. \& Conciauro, G. (2002). Dispersion characteristics of substrate integrated rectangular waveguide, IEEE Microwave and Wireless Components Letters, Vol.12, No.9, pp.333-335

Chadha, R. \& Gupta, K.C. (1981). Segmentation method using impedance matrices for analysis of planar microwave circuits, IEEE Transactions on Microwave Theory and Techniques, Vol.MTT-29, No.1, pp.71-74

Deslandes, D. \& Wu, K. (2005). Substrate integrated waveguide leaky-wave antenna: concept and design considerations, Proceedings of 2005 Asia-Pacific Microwave Conference, pp. 346-349, Dec. 2005

Hirokawa, J. \& Ando, M. (1998). Single-layer feed waveguide consisting of posts for plane TEM wave excitation in parallel plates, IEEE Transactions on Antennas and Propagation, Vol.46, No.5, pp. 625-630

Kishihara, M., Ohta, I. \& Yamane, K. (2006). Analysis of waveguide H-plane discontinuities with metallic/dielectric obstacles, IEICE Transactions on Electronics (Japanese Edition), Vol.J89-C, No.7, pp. 475-484

Kishihara, M., Yamane, K. \& Ohta, I. (2004). Analysis of H-plane waveguide discontinuities by segment substitution process, Proceedings of 2004 Asia Pacific Microwave Conference, B4.2, pp.196-197 in abstract book, Dec. 2004.

Moldovan, E., Bosisio, R.G. \& Wu, K. (2006). W-band multiport substrate-integrated waveguide circuits, IEEE Transactions on Microwave Theory and Techniques, Vol.54, No.2, pp. 625-632

Ohta, I., Toda K., Kishihara, M. \& Kawai, T. (2007). Design of cruciform substrate-integrated waveguide hybrids based on H-plane planar circuit approach, Proceedings of 2007 Asia-Pacific Microwave Conference, pp.683-686, Dec. 2007

Powell, M.J.D. (1964), An efficient method for finding the minimum of a function of several variables without calculating derivatives, Computer Journal, Vol.7, pp.155-162

Pozar, D.M. (1998). Microwave Engineering, Second Edition, John Wiley \& Sons, 0-471-17096-8

Toda, K., Ohta, I. \& Kishihara, M. (2006). H-plane crossed-waveguide hybrids, Proceedings of 36th European Microwave Conference, pp. 987-990, Sept. 2006

$\mathrm{Wu}, \mathrm{K}$. (2001). Integration and interconnect techniques of planar and non-planar structures for microwave and millimeter-wave circuits - Current status and future trend, Proceedings of 2001 Asia-Pacific Microwave Conference, pp. 411-416, Dec. 2001

$\mathrm{Xu}, \mathrm{X}$. , Bosisiso, R.G. \& Wu, K. (2005). A new six-port junction based on substrate integrated waveguide technology, IEEE Transactions on Microwave Theory and Techniques, Vol.53, No.7, pp. 2267-2273

$\mathrm{Xu}, \mathrm{F}$. \& Wu, K. (2005). Guided-wave and leakage characteristics of substrate integrated waveguide, IEEE Transactions on Microwave Theory and Techniques, Vol.53, No.1, pp. $66-73$ 


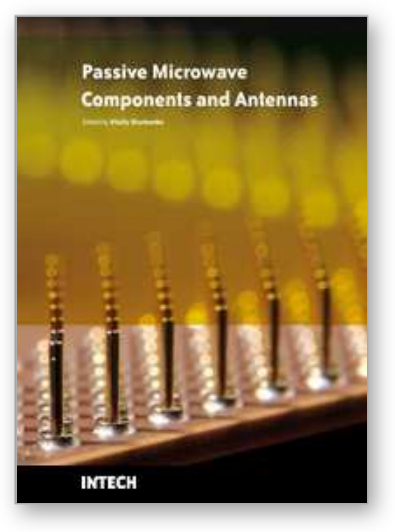

\author{
Passive Microwave Components and Antennas \\ Edited by Vitaliy Zhurbenko
}

ISBN 978-953-307-083-4

Hard cover, 556 pages

Publisher InTech

Published online 01, April, 2010

Published in print edition April, 2010

Modelling and computations in electromagnetics is a quite fast-growing research area. The recent interest in this field is caused by the increased demand for designing complex microwave components, modeling electromagnetic materials, and rapid increase in computational power for calculation of complex electromagnetic problems. The first part of this book is devoted to the advances in the analysis techniques such as method of moments, finite-difference time- domain method, boundary perturbation theory, Fourier analysis, mode-matching method, and analysis based on circuit theory. These techniques are considered with regard to several challenging technological applications such as those related to electrically large devices, scattering in layered structures, photonic crystals, and artificial materials. The second part of the book deals with waveguides, transmission lines and transitions. This includes microstrip lines (MSL), slot waveguides, substrate integrated waveguides (SIW), vertical transmission lines in multilayer media as well as MSL to SIW and MSL to slot line transitions.

\title{
How to reference
}

In order to correctly reference this scholarly work, feel free to copy and paste the following:

Isao Ohta and Mitsuyoshi Kishihara (2010). Analysis and Design of SIW Components Based on H-Plane Planar Circuit Approach, Passive Microwave Components and Antennas, Vitaliy Zhurbenko (Ed.), ISBN: 978953-307-083-4, InTech, Available from: http://www.intechopen.com/books/passive-microwave-componentsand-antennas/analysis-and-design-of-siw-components-based-on-h-plane-planar-circuit-approach

\section{INTECH}

open science | open minds

\section{InTech Europe}

University Campus STeP Ri

Slavka Krautzeka 83/A

51000 Rijeka, Croatia

Phone: +385 (51) 770447

Fax: +385 (51) 686166

www.intechopen.com

\section{InTech China}

Unit 405, Office Block, Hotel Equatorial Shanghai

No.65, Yan An Road (West), Shanghai, 200040, China

中国上海市延安西路65号上海国际贵都大饭店办公楼405单元

Phone: +86-21-62489820

Fax: $+86-21-62489821$ 
(C) 2010 The Author(s). Licensee IntechOpen. This chapter is distributed under the terms of the Creative Commons Attribution-NonCommercialShareAlike-3.0 License, which permits use, distribution and reproduction for non-commercial purposes, provided the original is properly cited and derivative works building on this content are distributed under the same license. 